\title{
Reversión de Esterilización Tubárica por la Técnica de Macrocirugía
}

Dr. Lyis Alfonso López Jiménez *
Dr. Jorge Enrique Corzo C. *

\section{INTRODUCCION}

El auge de la esterilización femenina en los últimos años, por diferentes métodos quirúrgicos, ha creado la necesidad de revertir dicha esterilización para brindar la posibilidad de un nuevo hijo a mujeres que así lo desean, generalmente motivadas por pérdida de hijos o nuevo matrimonio.

La cirugía tubárica para revertir la esterilización por la técnica de macrocirugía ha reportado en el pasado resultados desalentadores, dependiendo de la técnica usada $y$ de los cuidados pre $y$ postoperatorios (2). Para esta cirugía se han utilizado diferentes tipos de prótesis tubáricas. Los primeros informes en nuestra época fueron resultado de los trabajos de Hellman en 1951 (3). Posteriormente, (1956) Siegler y Hellman (5) muestran una serie de 191 anastomosis realizadas por 53 cirujanos con una rata de embarazo de $7.8 \%$, la mitad de las cuales $(3.9 \%)$ tuvieron parto a término. En la Tabla No. 1 se muestran los resultados obtenidos por diferentes autores con la técnica de macrocirugía tubárica.

(*) Especialistas del Depto. de Gineco-obstetricia del Hospital Militar Central.
El propósito de este artículo es informar los resultados obtenidos en pacientes a quienes se les practicó reversión de la esterilización, utilizando las técnicas de macrocirugía con algunas modificaciones a los cuidados pre y post-operatorios propuestos por Cognat (2).

\section{Material y métodos}

Se presentan los resultados obtenidos con reversión de esterilización tubárica en 4 pacientes intervenidas en el Hospital Militar Central, con técnicas de macrocirugía, durante el tiempo comprendido entre diciembre de 1978 y noviembre de 1979. Las características de las pacientes intervenidas se muestran en la Tabla No. 2

\section{Métodos}

Dividimos el trabajo para su presentación en tres partes:

a) Cuidados preoperatorios, b) Técnica quirúrgica $\vee$ c) cuidados post-operatorios.

\section{a. Cuidados Preoperatorios}

No utilizamos las hidrotubaciones recomendadas por Cognat (2) para evitar 
TABLA No. 1

Resultados publicados por diferentes autores sobre reversión de esterilización tubárica con la técnica de Macrocirugía

\begin{tabular}{|c|c|c|c|c|c|}
\hline \multirow{2}{*}{ Autor } & \multirow{2}{*}{$\begin{array}{l}\text { Método de Esteri- } \\
\text { lización. }\end{array}$} & \multirow{2}{*}{$\begin{array}{l}\text { Método de } \\
\text { Reversión }\end{array}$} & \multirow{2}{*}{ Casos } & \multicolumn{2}{|c|}{ EMBARAZO } \\
\hline & & & & Intrauterino & Ectópico \\
\hline $\begin{array}{l}\text { WILLIAMS } \\
\text { (1973) }\end{array}$ & Ligadura & Macrocirugía & 6 & $0(0 \%)$ & $0(0 \%)$ \\
\hline $\begin{array}{l}\text { UMEZAKI et al } \\
(1974)\end{array}$ & Ligadura & Macrocirugía & 6 & $3(50 \%)$ & $0(0 \%)$ \\
\hline SIEGLER y PEREZ & Ligadura & Macrocirugía & 17 & $4(24 \%)$ & $1(6 \%)$ \\
\hline $\begin{array}{l}\text { HODARI et al } \\
(1977)\end{array}$ & $\begin{array}{l}\text { Ligadura (12) } \\
\text { Cauterio (2) }\end{array}$ & Macrocirugía & 14 & $4(29 \%)$ & $3(21 \%)$ \\
\hline $\begin{array}{l}\text { WHEELESS } \\
(1977)\end{array}$ & Cauterio & Macrocirugía & 1 & $0(0 \%)$ & $0(0 \%)$ \\
\hline LOPEZ-CORZO & Ligadura & Macrocirugía & 4 & $1(25 \%)$ & $1(25 \%)$ \\
\hline
\end{tabular}

TABLA No. 2

Características de las pacientes del presente trabajo

\begin{tabular}{ccccccc}
\hline aso No. & Edad & $\begin{array}{c}\text { No. de hijos } \\
\text { vivos }\end{array}$ & $\begin{array}{c}\text { Tiempo entre la } \\
\text { esterilizacion } y \\
\text { reversión }\end{array}$ & $\begin{array}{l}\text { Indicaciones de la } \\
\text { esterilidad }\end{array}$ & $\begin{array}{l}\text { Técnica de la } \\
\text { esterilización }\end{array}$ & $\begin{array}{c}\text { Indicación de la } \\
\text { reversión }\end{array}$ \\
\hline 1 & 35 & 4 & $31 / 2$ años & $\begin{array}{l}\text { No deseaba más } \\
\text { hijos }\end{array}$ & Pomeroy & $\begin{array}{l}\text { Deseo de nuevo } \\
\text { hijo }\end{array}$ \\
2 & 30 & G5P5 $\left(^{*}\right)$ & 8 años & $\begin{array}{l}\text { No deseaba más } \\
\text { hijos } \\
\text { No deseaba más }\end{array}$ & Pomeroy & $\begin{array}{l}\text { No tiene hijos } \\
\text { hijos } \\
\text { niovo matrimo- }\end{array}$ \\
4 & 29 & 4 & 8 años & $\begin{array}{l}\text { No deseaba más Pomeroy } \\
\text { hijos }\end{array}$ & $\begin{array}{l}\text { Deseo de nuevo } \\
\text { hijo. }\end{array}$ \\
\hline
\end{tabular}

(*) Todos los hijos murieron en accidente después de la ligadura. 
cualquier irritación posible en el endosalpinx. Como cuidado preoperatorio se utilizaron 12 horas antes del acto operatorio las siguientes drogas: $32 \mathrm{mg}$ de Dexametasona por vía intramuscular, Penicilina cristalina 4 millones I. V. Y Estreptomicina $1 \mathrm{gr}$. I. M.

Todas las pacientes tenían Histerosalpingografía que demostraba la obstrucción tubárica y exámenes descartando los demás factores causantes de esterilidad.

\section{b. Técnica quirúrgica}

La técnica empleada consistió en anastomosis término-terminal a diferentes niveles según se muestra en la Tabla No. 3 . En todos los casos se utilizó un catéter de polietileno colocado a través de la trompa hasta la cavidad uterina y el otro extremo se fijó a piel en el borde de la incisión de Phannenstiel. Se hicieron pruebas de permeabilidad de la anastomosis usando Azul de Metileno inyectado a través del fondo uterino. Se utilizó irrigación con Dexametasona y Penicilina y se colocaron compre- sas en el fondo de saco de Douglas para elevar el útero y anexos. Se emplearon suturas de Prolene 6-0 que interesaban las capas seromuscular de la trompa y muscular uterina cuando se efectuó anasto. mosis tubo-cornual.

\section{c. Cuidados post-operatorios}

Se administraron $1 \mathrm{gr}$ de tetraciclina I. V. diario durante 3 días y luego $1 \mathrm{gr}$. diario por vía oral durante 30 días. Dexametazona $24 \mathrm{mg} \mathrm{I}$. M. diarios durante 3 dias, luego $1.5 \mathrm{mg}$ por vía oral durante 8 días, a continuación $1 \mathrm{mg}$ por vía oral durante 15 días y finalmente $0.5 \mathrm{mg}$. por vía oral durante 8 dias hasta el retiro del catéter de polietileno.

En el momento de retirar el catéter, es decir a los 30 días, se practicó la primera persuflación la cual se repitió en 3 ocasiones entre el 5o. y 8o. día del ciclo menstrual. Todas las persuflaciones indicaron permeabilidad tubárica y peristalsis satisfactoria. El catéter fue retirado el día 30 post-operatorio. En el caso No. 2 de nues-

TABLA No. 3

Técnica quirúrgica empleada (Macrocirugía)

\begin{tabular}{clcc}
\hline Caso No. & Anastomosis término - terminal & Catéter & Corticoides intraoperatorios \\
\hline 1 & $\begin{array}{l}\text { istmico - cornual derecha } \\
\text { istmico - ampular izquierda }\end{array}$ & 30 días & Sí \\
2 & $\begin{array}{l}\text { ístmico - istmico bilateral } \\
3\end{array}$ & 30 días & Sí \\
& $\begin{array}{l}\text { istmico - ampular izquierda } \\
\text { istmico - cornual derecha }\end{array}$ & 30 días & Si \\
& istmico - cornual bilateral & 30 días & Si \\
\hline
\end{tabular}


tro trabajo, el catéter de una trompa fue extraido accidentalmente al tercer día post-operatorio.

\section{Resultados}

De las cuatro pacientes se obtuvieron hasta la fecha 2 embarazos; uno intrauterino que llegó a término con feto vivo $y$ la paciente fue desembarazada con cesárea; en la revisión intraoperatoria las trompas se encontraron de características normales sin adherencias. La otra paciente presentó embarazo ectópico y fue laparotomizada practicándose salpingectomía; la trompa contralateral se encontró de características normales. Las otras dos pacientes tienen permeabilidad tubárica pero, no se han embarazado hasta la fecha. Esto representa una tasa de embarazo del $50 \%$, con un $25 \%$ de embarazo con feto vivo a término. Tabla No. 4

\section{Discusión}

Los porcentajes de éxito presentados por otros autores quienes no utilizaron prótesis post-operatorias por largo tiempo podrían compararse con los presentados en nuestro grupo de estudio, siendo en la mayoría menores. (Tabla No. 1), (1).

La utilización de drogas en el intra y post-operatorio, como los corticoides, es discutido por algunos autores debido al aumento de la tasa de infecciones $y$ adherencias, no se presentó en nuestra serie, siendo por el contrario favorable ya que en la segunda cirugía realizada en dos pacientes, no se encontraron adherencias. La utilización de catéter, por largo tiempo, no afectó la motilidad tubárica ni causó adherencias.

Las técnicas de microcirugía introducidas recientemente, dan porcentajes de éxito que superan en forma amplia los obtenidos con macrocirugía $(60 \%)$, Tabla No. 5 (1). Informes aún más recientes indican que este porcentaje de éxito ha aumentado hasta el $75 \%$. Esto implica que a pesar de los éxitos obtenidos con macrocirugía hay necesidad de utilizar las técnicas de microcirugía para nuevos casos de reversión de esterilización tubárica.

TABLA No. 4

\begin{tabular}{|c|c|c|c|c|}
\hline \multirow[t]{2}{*}{ Caso No. } & \multicolumn{2}{|c|}{ EMBARAZO } & \multirow{2}{*}{$\begin{array}{l}\text { Tiempo entre la } \\
\text { reversión y el em- } \\
\text { barazo }\end{array}$} & \multirow{2}{*}{$\begin{array}{c}\text { Permeabilidac } \\
\text { tubárica }\end{array}$} \\
\hline & Intrauterino & Ectópico & & \\
\hline 1 & $1(25 \%)$ & & 4 meses & Si \\
\hline 2 & & $1(25 \%)$ & 11 meses & Sí \\
\hline 3 & - & - & - & Sí \\
\hline 4 & - & - & - & Sí \\
\hline
\end{tabular}


TABLA No. 5

Resultados publicados de reversión de esterilización tubárica: comparación entre Macro y Microcirugía

\begin{tabular}{cccc}
\hline Método & Casos & $\begin{array}{c}\text { Embarazos } \\
\text { Intrauterinos }\end{array}$ & $\begin{array}{c}\text { Embarazos } \\
\text { Extrauterinos }\end{array}$ \\
\hline Macrocirugía & 44 & $11(25 \%)$ & $4(9 \%)$ \\
Microcirugía & 35 & $21 \mathrm{~m}(60 \%)$ & $2(6 \%)$ \\
\hline
\end{tabular}

\section{Resumen y conclusiones}

Se presenta una serie de reversión de esterilización post-ligadura de trompas de 4 pacientes intervenidas entre noviembre de 1978 y diciembre de 1979 utilizando las técnicas de macrocirugía con catéter, a largo plazo. Los resultados dan una rata de éxito del $50 \%$ de embarazos, con $25 \%$ intrauterinos, a término y feto vivo.
Representa un trabajo preliminar de reversión de esterilización, utilizando la macrocirugía en un pequeño grupo de pacientes. Dado que en la actualidad se está utilizando el método de reversión por microcirugía esperamos obtener mejores resultados. Queremos informar estos métodos de macrocirugía, que podrían ser de utilidad en los centros que no cuentan con microcirugía todavía.

\section{TUBAL STERILIZATION REVERSION BY MEANS OF THE MACROSURGERY TECHNIQUE}

A reversion of tubes post-ligature sterilization appeared in 4 patients operated between November 1978 and December 1979 applying both macrosurgery with catheter and long term corticosteroids. The results show a success rate of $50 \%$ completed and alive fetus pregnancies, with $25 \%$ intrauterine ones.

The use of Macrosurgery in a small group of patients represents a preliminary work in sterilization reversion. Since the reversion method by Macrosurgery is presently used, we tope to achieve better results.
We want to inform about these Macrosurgery methods, which could be usefull in those centers without Microsurgery.

\section{Bibliografía}

1. BERGER G. Reversal of Sterilization, Series on Fertility Regulation. New York, Harper and Row, 1978. pp. 170-76

2. COGNAT M. Enfoque actual del tratamiento quirúrgico de la esterilidad de origen tubárico. Clin. Ginecol. 3 (2): 138, 1978. 
3. HELLMAN LM. The use of Polythylene in human tubal plastic operations. Fertil Steril (2) 498, 1951.

4. GOMEL V. Microsurgery in gynecology, St. Louis, Christian Board of Publications, 1977. pp. 114-18.
5. SIEGLER AM. HELLMAN LM. Tubal plastic surgery. Fertil Steril; (7) 170 , 1956.

6. WINSTON R. Microsurgery in gynecology, St. Louis, Christian Board of Publications, 1977. pp. 135-37. 